\title{
Spatial inhomogeneity and the metal-insulator transition in $\mathrm{Ca}_{3}\left(\mathrm{Ru}_{1-x} \mathrm{Ti}_{x}\right)_{2} \mathrm{O}_{7}$
}

\author{
Frank Lechermann $\odot,{ }^{1}$ Qiang Han, ${ }^{2}$ and Andrew J. Millis ${ }^{2,3}$ \\ ${ }^{1}$ I. Institut für Theoretische Physik, Universität Hamburg, Jungiusstrasse 9, 20355 Hamburg, Germany \\ ${ }^{2}$ Department of Physics, Columbia University, 538 West 120th Street, New York, New York 10027, USA \\ ${ }^{3}$ Center for Computational Quantum Physics, The Flatiron Institute, 162 5th Avenue, New York, New York 10010, USA
}

(Received 11 June 2020; accepted 9 September 2020; published 24 September 2020)

\begin{abstract}
Turning a pristine Mott insulator into a correlated metal by chemical doping is a common procedure in strongly correlated materials physics, e.g., underlying the phenomenology of high- $T_{c}$ cuprates. The ruthenate bilayer compound $\mathrm{Ca}_{3} \mathrm{Ru}_{2} \mathrm{O}_{7}$ is a prominent example of a reversed case, namely, a correlated metal at stoichiometry that realizes a transition into an insulating state via Ti doping. We here investigate this puzzling metal-insulator transition (MIT) by first-principles many-body theory and elucidate a challenging interplay between electronic correlations and symmetry breaking on the $\mathrm{Ru}$ sublattice. While average effects on the $\mathrm{Ca}_{3} \mathrm{Ru}_{2} \mathrm{O}_{7}$ crystal structure are still relevant, the key to the MIT is the cooperation of electronic correlations with the spatial inhomogeneity in the defect regime. Together they give rise to the emergence of site-selective Mott criticality and competing orbital-ordering tendencies.
\end{abstract}

DOI: 10.1103/PhysRevResearch.2.033490

\section{INTRODUCTION}

The interplay of various degrees of freedom, e.g., of a charge, orbital, spin, or structural kind, is key to an understanding of many realistic metal-insulator transitions (MITs) in nature [1]. In this respect, the Ruddlesden-Popper series of $n$-layered calcium ruthenates $\mathrm{Ca}_{n+1} \mathrm{Ru}_{n} \mathrm{O}_{3 n+1}$ poses a particularly challenging problem. It is agreed that electronic correlations arise from low-spin $\mathrm{Ru}^{4+}\left(4 d^{4}\right)$ sites in these compounds, formally locating them in a Hund-metal [2-4] regime. While the $n \rightarrow \infty$ perovskite $\mathrm{CaRuO}_{3}$ is metallic [5] with competing magnetic interactions [6] down to the lowest temperatures, distorted $\mathrm{Ca}_{2} \mathrm{RuO}_{4}(n=1)$ undergoes a paramagnetic MIT at $T_{\mathrm{MIT}}=357 \mathrm{~K}$ and displays antiferromagnetic (AFM) order below $T_{N}=110 \mathrm{~K}$ [7]. From these limiting cases, Mott criticality is expectedly intricate in the bilayer system. Indeed, though $\mathrm{Ca}_{3} \mathrm{Ru}_{2} \mathrm{O}_{7}$ shows several electronic and magnetic transitions for $T<100 \mathrm{~K}$ [8-12], a robust insulating state is not reached at stoichiometry. With its noncentrosymmetric $B b 2_{1} m$ space group, the bilayer ruthenate marks the case of a polar metal [13-15] and has gained recent strong interest due to a complex fermiology [10,16-18].

Doping of about 5\% titanium gives rise to a MIT in the bilayer compound $[12,19]$ at $T_{\mathrm{MIT}} \sim 80 \mathrm{~K}$. The magnetic order switches from the stoichiometric A-type ordering of AFM-coupled ferromagnetic bilayers to G-type AFM (G-AFM) ordering. There is apparently no paramagnetic

Published by the American Physical Society under the terms of the Creative Commons Attribution 4.0 International license. Further distribution of this work must maintain attribution to the author(s) and the published article's title, journal citation, and DOI.
Mott-insulating phase in $\mathrm{Ca}_{3}\left(\mathrm{Ru}_{1-x} \mathrm{Ti}_{x}\right)_{2} \mathrm{O}_{7}$. Weakly localized states are already observed for very small Ti doping [20], and percolative behavior is also detected [12]. According to Ke et al. [19], the substitutional dopants enter as $\mathrm{Ti}^{4+}\left(3 d^{0}\right)$ impurities and therefore do not provide any significant charge doping. Hence the doping-induced MIT was originally associated with the blocking of hopping paths [19]. Furthermore, the averaged crystal structure of the Ti-doped bilayer [12] displays also an enhanced two-dimensionality of the bilayers as well as a somewhat increased tilting of the $\mathrm{RuO}_{6}$ octahedra. These global structural changes lead to a larger averaged crystal-field (CF) splitting $\Delta$ between the three partially occupied $\mathrm{Ru}(4 d)$ states $m=x y, x z / y z$ of $t_{2 g}$ character. In fact, a large $\Delta$ is the major driving force for the MIT in $\mathrm{Ca}_{2} \mathrm{RuO}_{4}$ $[21,22]$. Since the energy scales in $\mathrm{Ca}_{3} \mathrm{Ru}_{2} \mathrm{O}_{7}$ are generally smaller than in the latter single-layer ruthenate, the competition between the various degrees of freedom is much more subtle.

In this work, the goal is to unveil the detailed cooperation of defect physics and electronic correlations that drive the MIT in Ti-doped $\mathrm{Ca}_{3} \mathrm{Ru}_{2} \mathrm{O}_{7}$. By means of the real-space combination of density functional theory (DFT) and dynamical mean-field theory (DMFT) applied to a defect supercell with $6.25 \% \mathrm{Ti}$ concentration in a fully charge self-consistent manner, we profoundly account for average and local effects on an equal footing. While average effects from doping are notable, the spatial inhomogeneity introduced by $\mathrm{Ti}$ defects and cooperating with electron correlations is the crucial driving force towards the insulating phase.

\section{THEORETICAL APPROACH}

Charge self-consistent DFT + DMFT [23-25] is used to access the correlated electronic structure. For the DFT part, a mixed-basis pseudopotential method [26,27], employing 
the generalized-gradient approximation in the Perdew-BurkeErnzerhof form [28], is put into practice. Spin-orbit coupling is neglected in this work. The multiple single-site DMFT impurity problems encountered in the basic unit cell as well as in the defect supercell are solved by the hybridization-expansion continuous-time quantum Monte Carlo scheme [29], as implemented in the TRIQS code [30,31]. The correlated subspace consists of the effective transition-metal (TM) $t_{2 g}$ Wannierlike functions $w_{m}\left(t_{2 g}\right)$, i.e., covers locally three orbitals. These functions are obtained from the projected-local-orbital formalism [32,33], using as projection functions the linear combinations of atomiclike $t_{2 g}$ orbitals that diagonalize the TM local $w_{m}\left(t_{2 g}\right)$-orbital density matrix on each site.

Local Coulomb interactions in general Slater-Kanamori form, i.e., including density-density as well as spin-flip and pair-hopping terms, are parametrized by a Hubbard $U$ and a Hund's exchange $J_{\mathrm{H}}$. The intraorbital interaction $U$ on the Ru sites is treated as a parameter, ranging at most from $1.5 \mathrm{eV}$ to $7.5 \mathrm{eV}$. The Hund's exchange is chosen to be $J_{\mathrm{H}}=0.4 \mathrm{eV}$ for $U<2 \mathrm{eV}$ and $J_{\mathrm{H}}=0.7 \mathrm{eV}$ for $U>2 \mathrm{eV}$, in order to allow for a comparison with previous theoretical work on ruthenates [21]. For the Ti sites, a value of $U=5 \mathrm{eV}$ (and $J_{\mathrm{H}}=0.7 \mathrm{eV}$ ) is chosen to account for the fact that interactions in the $\operatorname{Ti}(3 d)$ shell may be larger than in the $\mathrm{Ru}(4 d)$ shell. To obtain the spectral information, analytical continuation from Matsubara space by the maximum-entropy method and the Padé method is performed.

\section{STOICHIOMETRIC $\mathrm{Ca}_{3} \mathrm{Ru}_{2} \mathrm{O}_{7}$}

To set the stage, we start with the correlated electronic structure of stoichiometric $\mathrm{Ca}_{3} \mathrm{Ru}_{2} \mathrm{O}_{7}$. Figure 1(a) displays the experimental unit cell [13] at $40 \mathrm{~K}$ with in-plane lattice parameters $a=5.37 \AA, b=5.54 \AA$, and $c=19.52 \AA$. It incorporates two bilayers along the $c$ axis and a total of eight $\mathrm{Ru}$ sites. The relevant CF splitting $\Delta$ in the $t_{2 g}$ manifold of the $\mathrm{Ru}(4 d)$ states is defined as $\Delta=\varepsilon_{x y}-\left(\varepsilon_{x z}+\varepsilon_{y z}\right) / 2$, where $\varepsilon_{m}$ are the CF levels of the $x y, x z$, and $y z$ Wannier orbitals. The effects due to the small splitting between $x z$ and $y z$ states are minor and will not be discussed henceforth (though the corresponding splitting is surely included in the calculations). On the DFT level, the bare CF splitting amounts to $\Delta=-153 \mathrm{meV}$, i.e., the $x y$ state is lowest in energy. This results in an orbital polarization, giving rise to occupations $n_{x y}=1.43$ and $n_{x z / y z}=1.26$ per orbital. Note that the given CF splitting is about half the value of $\Delta \sim-320 \mathrm{meV}$ in single-layer $\mathrm{Ca}_{2} \mathrm{RuO}_{4}$ [21].

The correlated spectral data at $T=100 \mathrm{~K}$ are depicted in Figs. 1(b)-1(d). The compound is metallic, but compared to DFT, strong renormalization and loss of coherence are revealed at low energy, in accordance with angle-resolved photoemission $[10,16,17]$. Significant in-plane anisotropy in the correlated fermiology takes place between $M_{\mathrm{a}}$ and $M_{\mathrm{b}}$, in line with experiment [10]. A more thorough investigation of further lower-temperature dispersions requires the inclusion of spin-orbit coupling and is not an objective of the present study. Finally, the local spectral functions show that the $x z / y z$. states are more strongly correlated with a lower Hubbard band of enhanced weight and deeper energy location of $\sim-2.9 \mathrm{eV}$ than the $x y$ states.

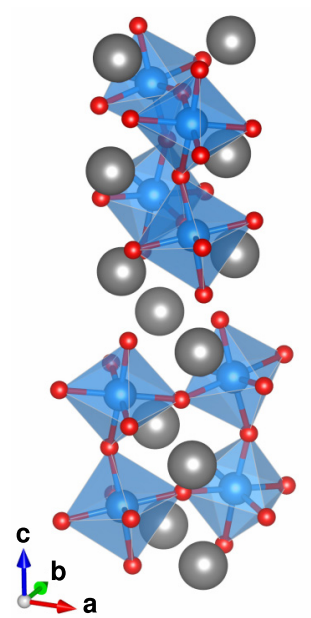

(a)

FIG. 1. Characterization of stoichiometric $\mathrm{Ca}_{3} \mathrm{Ru}_{2} \mathrm{O}_{7}$. (a) Unit cell with two bilayers: $\mathrm{Ca}$ (large gray), $\mathrm{Ru}$ (blue), and $\mathrm{O}$ (small red). (b)-(d) Paramagnetic DFT + DMFT results for $U=4.25 \mathrm{eV}$ at $T=100 \mathrm{~K}$. (b) Spectral function $A(\mathbf{k}, \omega)$ along high-symmetry lines, compared to DFT bands (green). (c) Fermi surface in the $k_{z}=0$ plane. The gray square marks the Brillouin zone. (d) Local $\mathrm{Ru} t_{2 g}$ spectral function discriminating the $x y$ and $x z / y z$ orbitals and comparing to the DFT spectrum.

\section{LOCAL ELECTRONIC STRUCTURE OF $\mathrm{Ca}_{3}\left(\mathrm{Ru}_{0.9375} \mathrm{Ti}_{0.0625}\right)_{2} \mathrm{O}_{7}$}

In order to describe the bilayer ruthenate with finite Ti doping, we start from the experimentally averaged (EA) structure at $10 \mathrm{~K}$ with $5 \%$ Ti doping [19]. The corresponding experimental system is insulating below $T_{\mathrm{MIT}} \sim 80 \mathrm{~K}$. As an averaged structure, the atom-number size of the primitive cell is identical to the one at stoichiometry, but with different lattice parameters $a=5.38 \AA, b=5.56 \AA$, and $c=19.37 \AA$, as well as modified atomic positions. Thus the effect of $\mathrm{Ti}$ impurities is only taken into account implicitly via an averaged structure modification. The effective $\mathrm{CF}$ splitting in this structure amounts to $\Delta=-171 \mathrm{eV}$ in DFT, and hence is about $20 \mathrm{meV}$ larger than in the stoichiometric case. This is mainly attributed to an enhanced two-dimensionality and increased tilting of the $\mathrm{RuO}_{6}$ octahedra. It can be seen from the dashed lines in Fig. 2(b) that this treatment of Ti doping is not sufficient to render the system insulating for a reasonable 

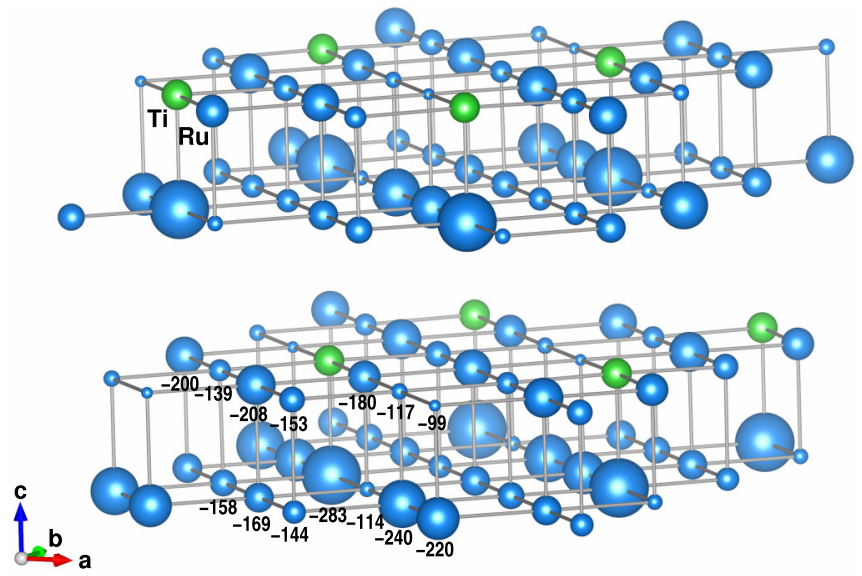

(a)

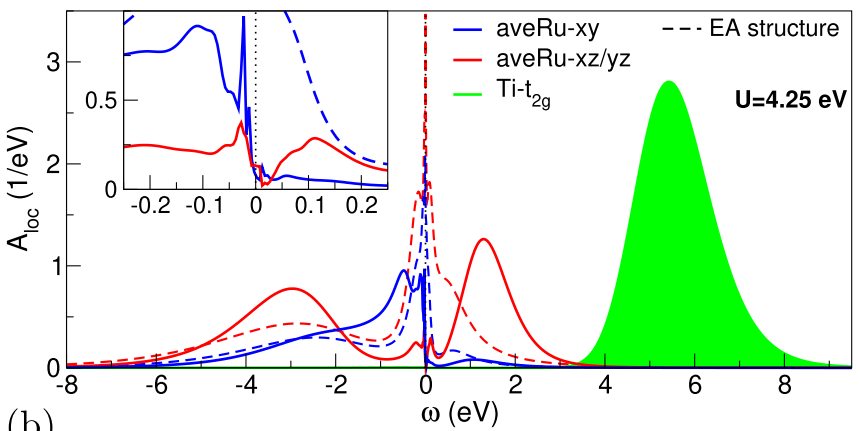

(b)

FIG. 2. Structure and global spectrum of $\mathrm{Ca}_{3}\left(\mathrm{Ru}_{0.9375} \mathrm{Ti}_{0.0625}\right)_{2} \mathrm{O}_{7}$. (a) Structurally relaxed supercell showing $\mathrm{Ru}$ (blue) and $\mathrm{Ti}$ (green) ions, indicating the crystal-field splitting $\Delta$ via the diameter of the $\mathrm{Ru}$-site spheres ( $\mathrm{Ca}$ and $\mathrm{O}$ sites are not shown). Numbers indicate the actual $\Delta$ value of a given Ru-site symmetry class (in meV). The diameter of the Ti spheres is chosen as the average $\Delta$ of the Ru sites. (b) Average $\mathrm{Ru}(4 d) t_{2 g}$ and $\mathrm{Ti}(3 d) t_{2 g}$ paramagnetic local spectrum with $6.25 \% \mathrm{Ti}$ for $U=4.25 \mathrm{eV}$ $(T=40 \mathrm{~K})$. Dashed lines show the corresponding $\mathrm{Ru} t_{2 g}$ spectrum of the EA structure for comparison. The inset shows a close-up of the low-energy region.

value of $U=4.25 \mathrm{eV}$, but electronic correlations are somewhat enhanced compared to the stoichiometric case.

Let us turn to the supercell description of Ti-doped $\mathrm{Ca}_{3} \mathrm{Ru}_{2} \mathrm{O}_{7}$. A 192-atom-site cell [cf. Fig. 2(a)] is constructed, starting from the EA structure and introducing two Ti impurities in adjacent bilayers, i.e., each bilayer carries one substitutional $\mathrm{Ti}$ defect. There are $32 \mathrm{TM}$ sites in the defect supercell, 30 of the $\mathrm{Ru}$ and 2 of the Ti kind. Fixing the scaled EA lattice parameters, we structurally relax this supercell within DFT $+\mathrm{U}$, assuming G-type AFM order. No symmetry constraints are enforced in the structural relaxation. This leads to site-symmetry breaking, not unexpected in this puzzling polar-metal system [34]. For the DFT + DMFT investigation at a system temperature of $T=40 \mathrm{~K}$, we identify $14 \mathrm{Ru}$-site classes as symmetry inequivalent. Together with both symmetry-equivalent Ti sites, there are hence 15 coupled impurity problems solved at each self-consistency step.

As in previous DFT calculations [19], the Ti impurities are indeed of the $\mathrm{Ti}^{4+}\left(3 d^{0}\right)$ kind, and the $t_{2 g}$ electronic

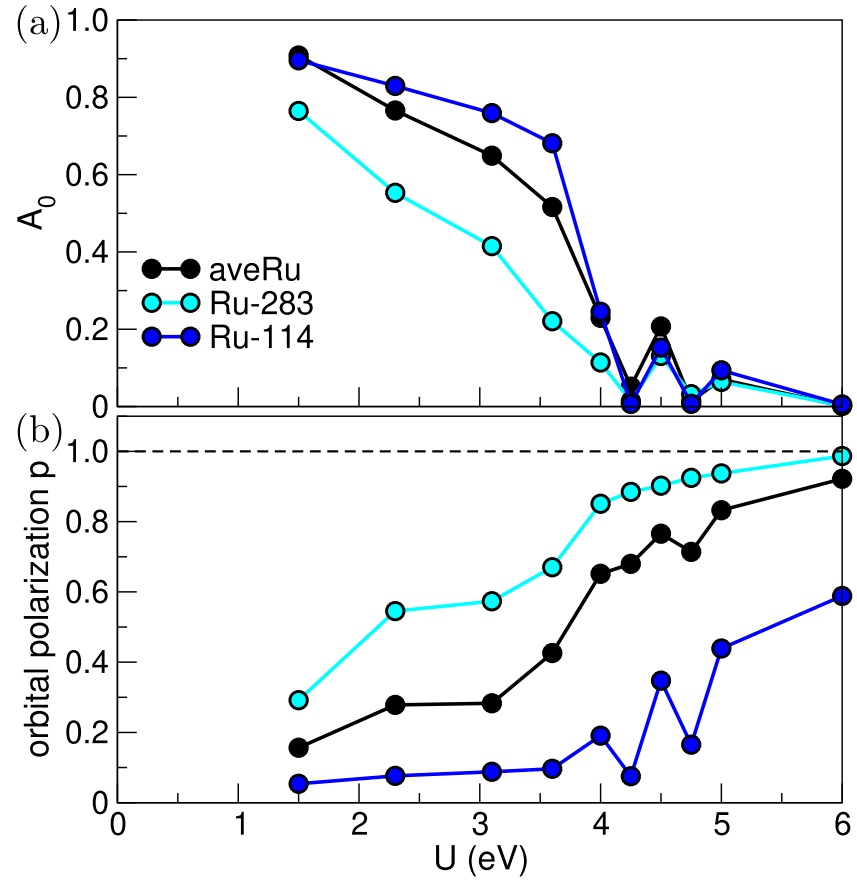

FIG. 3. (a) The $\mathrm{Ru}(4 d) t_{2 g}$ spectral weight $A_{0}$ at the Fermi level and (b) the orbital polarization $p$ with increasing $U$, for selected $\mathrm{Ru}$-site classes in the Ti-doped supercell, respectively, at $T=40 \mathrm{~K}$.

spectrum is accordingly located high in energy within the unoccupied region [see the green part in Fig. 2(b)]. Note that local structural relaxation shifts the Ti spectrum to somewhat smaller energies. The resulting CF splitting on the Ru sites is however distributed over a surprisingly large energy window $[-99,-283] \mathrm{eV}$. In order to simplify notation, we will in the following refer to the different Ru-site classes as $\mathrm{Ru} \Delta$. Interestingly, the Ru sites just below the Ti impurities have the largest CF splitting. These Ru-283 sites show a comparatively large relaxation away from Ti. The latter may be explained by the fact that due to the $3 d^{0}$ character of titanium, the effective $x z / y z$ hopping perpendicular to the plane is strongly weakened, resulting also in an overall reduced Ru-Ti bonding. Hence, the original effect of $\mathrm{Ti}$ sites blocking hopping paths is affirmed; however, this single-particle-based mechanism alone cannot be sufficient to drive the MIT at the given comparatively small amounts of doping.

Notably, the average DFT crystal-field splitting in the supercell amounts to $\Delta_{\mathrm{av}}=-172 \mathrm{eV}$ and is thus indeed identical to the one in the starting EA structure. However, for equal local Coulomb interactions, the system is Mott insulating with strong orbital polarization as shown in Fig. 2(b). On average, the $x y$ state becomes fully occupied and the $x z / y z$ states each host one electron such that the four-electron occupation of $\mathrm{Ru}(4 d) t_{2 g}$ is realized. This orbital-polarization scenario is reminiscent of the one in single-layer $\mathrm{Ca}_{2} \mathrm{RuO}_{4}$ [21]. However, since the average CF splittings of the EA structure and the aligned supercell correspond to each other, the spatial inhomogeneity has to play a key role in the bilayer MIT.

The site-selective data shown in Fig. 3 and corresponding electronic spectra in Fig. 4 render indeed obvious that the various $\mathrm{Ru}$ sites behave quite differently, in connection with their 


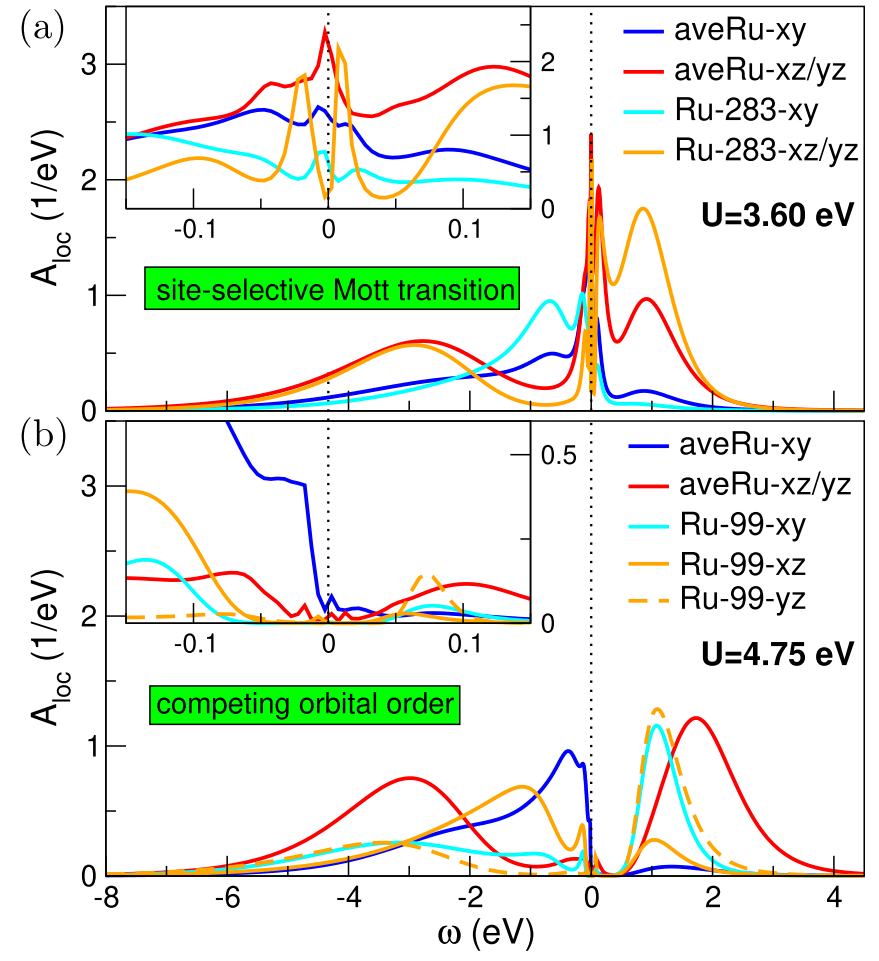

FIG. 4. Local $\mathrm{Ru}(4 d) t_{2 g}$ spectral function for (a) $U=3.60 \mathrm{eV}$ and (b) $U=4.75 \mathrm{eV}$ at $T=40 \mathrm{~K}$. The average spectra and two selected Ru-site class spectra are shown, respectively, for Ru-283 (Ru-99) at the lower (higher) $U$ value.

respective $\Delta$ values. In order to not only rely on analytical continuation, the $\mathrm{Ru}$ local spectral weight at lowest energy, i.e., $A_{\text {loc }}(\omega)$ at zero frequency $\omega=0$, is plotted in Fig. 3(a) in its approximate form $A_{0}=-\frac{\beta}{\pi} \sum_{m} \operatorname{Im} G_{\text {loc }}^{(m)}(\beta / 2)$, where $G_{\mathrm{loc}}^{m}(\tau)$ is the local one-particle Green's function for orbital $m$ and imaginary times $\tau$ at inverse temperature $\beta=1 / T$. The Ru-283 sites are much more strongly correlated than, e.g., the Ru-99 or Ru-114 sites with small $\Delta$. In fact, for $U=3.6 \mathrm{eV}$ the former sites have already gapped $\mathrm{Ru} t_{2 g}$ states, while on average the $\mathrm{Ru}$ sublattice still shows a metallic response [see the inset in Fig. 4(a)]. The wide spectrum of CF values based on the significant spatial inhomogeneity introduced by Ti doping therefore gives rise to a site-selective Mott scenario $[35,36]$ in $\mathrm{Ca}_{3}\left(\mathrm{Ru}_{0.9375} \mathrm{Ti}_{0.0625}\right)_{2} \mathrm{O}_{7}$. It occurs on the $\mathrm{Ru}$ sites perpendicular adjacent to the $\mathrm{Ti}$ impurities and is precursory to the MIT of the complete system at $U \sim 4 \mathrm{eV}$.

Not surprisingly, the orbital polarization, here simply defined as $p=n_{x y}-\left(n_{x z}+n_{y z}\right) / 2$, is much weaker for small- $\Delta$ sites. Those sites also cause site-selective physics, namely, an oscillatory-in- $U$ revival of metallicity for $U>4 \mathrm{eV}$. Only for $U=6 \mathrm{eV}$, all sites behave insulating in line. The given intermediate large- $U$ regime can be traced back to strong orbital(-order) competition originating from the small- $\Delta \mathrm{Ru}$ sites. On average, close to the metal-insulator transition, the $x y$ orbital becomes completely filled with two electrons, whereas the $x z$ and $y z$ orbitals become half filled, resulting in $p=1$. However, the Ru-99 sites, for example, mark a dominant $x z$ and close to half-filled $y z$ or $x y$ orbital filling for $U=4.75 \mathrm{eV}$ [see Fig. 4(b)].

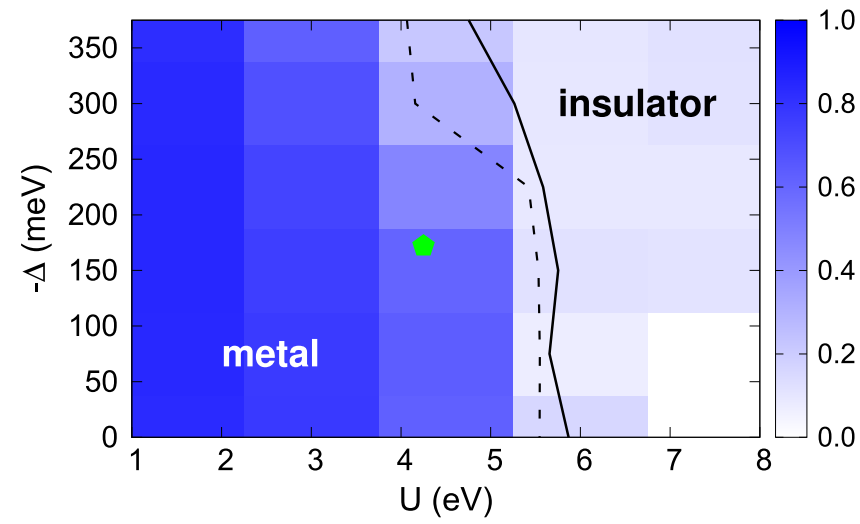

FIG. 5. Crystal-field vs $U$ phase diagram for Ti-doped $\mathrm{Ca}_{3} \mathrm{Ru}_{2} \mathrm{O}_{7}$ based on the EA structure at $T=100 \mathrm{~K}$ (see the text for further details). The blue color scale amounts to $A_{0}$ of $\mathrm{Ru}(4 d) t_{2 g}$ in the PM regime. Solid (Dashed) line marks the interpolated metal-insulator phase boundary, here roughly defined by $A_{0}=0.2$, in the PM (G-AFM) regime. The green pentagon marks the position of the onset of the insulating phase in the supercell calculations (here located via its average $\Delta$ value)

In order to render the comparison between the spatial homogeneous and inhomogeneous case more explicit, Fig. 5 displays the $-\Delta$ vs $U$ phase diagram of effective Ti-doped $\mathrm{Ca}_{3} \mathrm{Ru}_{2} \mathrm{O}_{7}$. It is constructed by utilizing the EA structure and gradually fixing $\Delta$ within the DFT + DMFT calculations. Note that charge self-consistency is an important ingredient, since orbital polarization and MIT tendencies depend thereon [37]. Since the true experimental system is AFM ordered in the insulating phase, results for G-AFM order of the EA structure are included. Those were obtained by enabling a spin-polarized self-energy in the DFT + DMFT cycle. As expected, the MIT occurs at somewhat smaller $U$ with G-AFM order, yet the net effect of magnetic ordering on the Mott criticality is not decisive. Increasing the absolute value of $\Delta$ fosters the driving toward the MIT, but the slope remains steep for $-\Delta<200 \mathrm{meV}$. The MIT occurs for moderate $U$ values only for rather large $\mathrm{CF}$ splittings. Note, in this respect, that a paramagnetic MIT was realized for single-layer $\mathrm{Ca}_{2} \mathrm{RuO}_{4}$ with $\Delta \sim-320 \mathrm{meV}$ for $U=3.1 \mathrm{eV}$ in a previous one-shot DFT + DMFT calculation [21]. However, such one-shot approaches are known for two features compared to charge self-consistent DFT + DMFT [37,38], namely, tending to overestimate orbital polarizations and often realizing smaller critical $U$ values. Furthermore, the MIT value of $U=4.25 \mathrm{eV}$ for the defect supercell (see the green pentagon in Fig. 5) is still well in the metallic region of the EA-based phase diagram. However, recall that the orbital-competing regime up to a robust spatially coherent insulating state extends also up to $U=6 \mathrm{eV}$ in the supercell calculations. Still, it may be inferred that spatial inhomogeneity, which is not included in the EA structure, is proactive in driving the MIT toward smaller $U$ values.

We moreover performed DFT + DMFT calculations, allowing for AFM ordering in the Ti-doped supercell. There also competing orderings, now between different AFM tendencies, occur: While for moderate $3<U<4 \mathrm{eV}$ the system tends to A-type-like ordering, reminiscent of the 
stoichiometric magnetic order, for $U>4 \mathrm{eV}$ indeed G-typelike ordering becomes more favorable. Above $U=6 \mathrm{eV}$ a very robust G-AFM ordering pattern is established.

\section{SUMMARY}

By means of large-scale first-principles many-body calculations, we showed that the MIT in Ti-doped $\mathrm{Ca}_{3} \mathrm{Ru}_{2} \mathrm{O}_{7}$ is mainly driven by the interplay of spatial inhomogeneity features and strong electronic correlations. Introduction of Ti impurities leads to a substantial energy spread of the $\mathrm{CF}$ splitting $\Delta$ across the Ru sublattice, resulting in siteselective Mott transitions for large- $\Delta$ sites and competitions in the orbital-ordering tendencies on small- $\Delta$ sites. This not only explains the occurrence of a doping-induced metalinsulator transition for reasonable interaction strengths, but accounts furthermore for the findings of percolative behavior. Other effects such as hopping blocking or average structural modification via a change of lattice parameters furthermore support the insulating tendencies with doping, but appear not decisive. The site-selective physics reported here may also be relevant for an understanding and modeling of recently discovered current-induced diamagnetism [39] in Ti-doped $\mathrm{Ca}_{3} \mathrm{Ru}_{2} \mathrm{O}_{7}$. Describing the transport scenario from a possibly current-induced (partial-)gap closing and/or the coexistence of diamagnetism with persisting (local) magnetic order could rely on the important interplay of electronic correlation and spatial inhomogeneity. Furthermore, concerning experiments, scanning-tunneling studies of $\mathrm{Ca}_{3}\left(\mathrm{Ru}_{1-x} \mathrm{Ti}_{x}\right)_{2} \mathrm{O}_{7}$ would be highly interesting to verify the site-dependent Mott physics revealed here.

Let us finally emphasize that sole averaged-structure investigations of defect problems in correlated materials may have their shortcomings. In a recent realistic DMFT study on impurities in $\mathrm{V}_{2} \mathrm{O}_{3}$ [37], it was shown that local pointgroup symmetry breaking from trigonal to monoclinic is essential to understand the Cr-induced paramagnetic MIT. Here Ti-induced site-symmetry breaking is key to the MIT in $\mathrm{Ca}_{3} \mathrm{Ru}_{2} \mathrm{O}_{7}$. Both results corroborate that the explicit and detailed cooperation of defect chemistry and many-body physics is at the heart of various doping problems in correlated matter.

\section{ACKNOWLEDGMENTS}

F.L. acknowledges financial support from the German Science Foundation via Project No. LE-2446/4-1. A.J.M. acknowledges support from the Basic Energy Sciences program of the US Department of Energy under grant SC-0018218. Computations were performed at the University of Hamburg and the JUWELS Cluster of the Jülich Supercomputing Centre under Project No. hhh08.
[1] Q. Han and A. Millis, Phys. Rev. Lett. 121, 067601 (2018),

[2] P. Werner, E. Gull, M. Troyer, and A. J. Millis, Phys. Rev. Lett. 101, 166405 (2008).

[3] K. Haule and G. Kotliar, New J. Phys. 11, 025021 (2009).

[4] L. de Medici, J. Mravlje, and A. Georges, Phys. Rev. Lett. 107, 256401 (2011).

[5] Q. Han, H. T. Dang, and A. J. Millis, Phys. Rev. B 93, 155103 (2016).

[6] T. He and R. J. Cava, Phys. Rev. B 63, 172403 (2001).

[7] C. S. Alexander, G. Cao, V. Dobrosavljevic, S. McCall, J. E. Crow, E. Lochner, and R. P. Guertin, Phys. Rev. B 60, R8422 (1999).

[8] G. Cao, S. McCall, J. E. Crow, and R. P. Guertin, Phys. Rev. Lett. 78, 1751 (1997).

[9] Y. Yoshida, I. Nagai, S.-I. Ikeda, N. Shirakawa, M. Kosaka, and N. Môri, Phys. Rev. B 69, 220411(R) (2004).

[10] F. Baumberger, N. J. C. Ingle, N. Kikugawa, M. A. Hossain, W. Meevasana, R. S. Perry, K. M. Shen, D. H. Lu, A. Damascelli, A. Rost, A. P. Mackenzie, Z. Hussain, and Z.-X. Shen, Phys. Rev. Lett. 96, 107601 (2006).

[11] J. S. Lee, S. J. Moon, B. J. Yang, J. Yu, U. Schade, Y. Yoshida, S.-I. Ikeda, and T. W. Noh, Phys. Rev. Lett. 98, 097403 (2007).

[12] J. Peng, X. Ke, G. Wang, J. E. Ortmann, D. Fobes, T. Hong, W. Tian, X. Wu, and Z. Q. Mao, Phys. Rev. B 87, 085125 (2013).

[13] Y. Yoshida, S.-I. Ikeda, H. Matsuhata, N. Shirakawa, C. H. Lee, and S. Katano, Phys. Rev. B 72, 054412 (2005).

[14] D. J. Singh and S. Auluck, Phys. Rev. Lett. 96, 097203 (2006).

[15] F. Thöle and N. A. Spaldin, Philos. Trans. R. Soc. A 376, 20170450 (2018).

[16] M. Horio, Q. Wang, V. Granata, K. P. Kramer, Y. Sassa, S. Jöhr, D. Sutter, A. Bold, L. Das, Y. Xu et al., arXiv:1911.12163.
[17] I. Marković, M. D. Watson, O. J. Clark, F. Mazzola, E. A. Morales, C. A. Hooley, H. Rosner, C. M. Polley, T. Balasubramanian, S. Mukherjee, N. Kikugawa, D. A. Sokolov, A. P. Mackenzie, and P. D. C. King, PNAS 117, 15524 (2020).

[18] D. Puggioni, M. Horio, J. Chang, and J. M. Rondinelli, Phys. Rev. Res. 2, 023141 (2020).

[19] X. Ke, J. Peng, D. J. Singh, T. Hong, W. Tian, C. R. Dela Cruz, and Z. Q. Mao, Phys. Rev. B 84, 201102(R) (2011).

[20] S. Tsuda, N. Kikugawa, K. Sugii, S. Uji, S. Ueda, M. Nishio, and Y. Maeno, Phys. Rev. B 87, 241107(R) (2013).

[21] E. Gorelov, M. Karolak, T. O. Wehling, F. Lechermann, A. I. Lichtenstein, and E. Pavarini, Phys. Rev. Lett. 104, 226401 (2010).

[22] A. Liebsch and H. Ishida, Phys. Rev. Lett. 98, 216403 (2007).

[23] S. Y. Savrasov, G. Kotliar, and E. Abrahams, Nature (London) 410, 793 (2001).

[24] L. V. Pourovskii, B. Amadon, S. Biermann, and A. Georges, Phys. Rev. B 76, 235101 (2007).

[25] D. Grieger, C. Piefke, O. E. Peil, and F. Lechermann, Phys. Rev. B 86, 155121 (2012).

[26] F. Lechermann, F. Welsch, C. Elsässer, C. Ederer, M. Fähnle, J. M. Sanchez, and B. Meyer, Phys. Rev. B 65, 132104 (2002).

[27] B. Meyer, C. Elsässer, F. Lechermann, and M. Fähnle, FORTRAN90 program for mixed-basis pseudopotential calculations for crystals, Max-Planck-Institut für Metallforschung, 1998 (unpublished).

[28] J. P. Perdew, K. Burke, and M. Ernzerhof, Phys. Rev. Lett. 77, 3865 (1996).

[29] P. Werner, A. Comanac, L. de' Medici, M. Troyer, and A. J. Millis, Phys. Rev. Lett. 97, 076405 (2006). 
[30] O. Parcollet, M. Ferrero, T. Ayral, H. Hafermann, I. Krivenko, L. Messio, and P. Seth, Comput. Phys. Commun. 196, 398 (2015).

[31] P. Seth, I. Krivenko, M. Ferrero, and O. Parcollet, Comput. Phys. Commun. 200, 274 (2016).

[32] B. Amadon, F. Lechermann, A. Georges, F. Jollet, T. O. Wehling, and A. I. Lichtenstein, Phys. Rev. B 77, 205112 (2008).

[33] V. I. Anisimov, D. E. Kondakov, A. V. Kozhevnikov, I. A. Nekrasov, Z. V. Pchelkina, J. W. Allen, S.-K. Mo, H.-D. Kim, P. Metcalf, S. Suga, A. Sekiyama, G. Keller, I. Leonov, X. Ren, and D. Vollhardt, Phys. Rev. B 71, 125119 (2005).
[34] A. Gangshettiwar, Y. Zhu, Z. Jiang, J. Peng, Y. Wang, J. He, J. Zhou, Z. Mao, and K. Lai, Phys. Rev. B 101, 201106(R) (2020).

[35] H. Park, A. J. Millis, and C. A. Marianetti, Phys. Rev. Lett. 109, 156402 (2012).

[36] F. Lechermann and M. Obermeyer, New J. Phys. 17, 043026 (2015).

[37] F. Lechermann, N. Bernstein, I. I. Mazin, and R. Valentí, Phys. Rev. Lett. 121, 106401 (2018).

[38] A. Hampel, S. Beck, and C. Ederer, Phys. Rev. Res. 2, 033088 (2020).

[39] C. Sow, R. Numasaki, G. Mattoni, S. Yonezawa, N. Kikugawa, S. Uji, and Y. Maeno, Phys. Rev. Lett. 122, 196602 (2019). 